\title{
In Situ Correlative Microscopy Combining Transmission Electron Microscopy and Secondary Ion Mass Spectrometry
}

Santhana Eswara ${ }^{1}$, Lluís Yedra ${ }^{1,2}$, Alisa Pshenova ${ }^{1}$, Varun Sarbada ${ }^{3}$, Jean-Nicolas Audinot ${ }^{1}$, Robert Hull $^{3}$ and Tom Wirtz ${ }^{1}$

1. Advanced Instrumentation for Ion Nano-Analytics (AINA), MRT Dept., Luxembourg Institute of Science and Technology, Belvaux, Luxembourg.

2. CentraleSupelec, France.

${ }^{3 .}$ Dept. of Materials Science and Engineering, Rensselaer Polytechnic Institute, Troy, NY.

Transmission Electron Microscopy (TEM) is well-known for high resolution imaging down to the atomic scale. The conventional analytical tools associated with TEM such as Energy Dispersive X-ray Spectroscopy (EDX) and Electron Energy-Loss Spectroscopy (EELS) are very powerful techniques for chemical imaging. However, they are inadequate for the characterization of trace elements (concentration $<0.1$ at. \%) and isotopes. On the other hand, Secondary Ion Mass Spectrometry (SIMS) is a high-sensitivity technique suitable to image even very low concentrations down to the ppm range. However, the SIMS image resolution is fundamentally limited by the ion-solid interaction volume to $\sim 10 \mathrm{~nm}$, although in practice, resolution in the range of $\sim 50 \mathrm{~nm}$ is typical. Recently high-brightness ion sources have enabled to push the SIMS image resolution down to $\sim 15 \mathrm{~nm}$. While impressive, it is still 2 to 3 orders of magnitude poorer in comparison to TEM. Considering the complementary strengths of the TEM and SIMS, we have developed a correlative microscopy tool combining these two techniques in situ $[1,2]$.

A FEI Tecnai F20 S/TEM with TWIN objective lens was modified such that a FEI FIB Magnum $\mathrm{Ga}^{+}$ ion-beam column and SIMS extraction could be integrated around the sample holder area. A compact magnetic-sector mass spectrometer was completely designed and developed in-house. A special highvoltage sample holder which could be biased up to $\pm 4.5 \mathrm{kV}$ was also developed to carry out in-situ SIMS analyses. With the sample holder tilted to $-68^{\circ}$, the FIB is oriented at $45^{\circ}$ to the sample surface and the SIMS extraction is aligned perpendicular to the sample surface. The configuration is illustrated in Figure 1. The TEM modification is such that all the standard imaging modalities of the TEM and STEM can be carried out without any restriction. During the SIMS imaging mode, the objective lens of the TEM is turned off in order to ensure that the ion beam trajectory is unaffected. An initial proof of concept was demonstrated using isotopically labelled lithium carbonate nanoparticles. Nanoparticles were identified using the isotopic labels $\left({ }^{6} \mathrm{Li}\right.$ and $\left.{ }^{7} \mathrm{Li}\right)$ and corresponding TEM images were acquired at higher resolution [2].

As SIMS signal intensity depends on the matrix elements (i.e. matrix effect), quantification of SIMS is difficult. As EDX is available in most electron microscopes, we have developed a quantification protocol in which SIMS quantification is guided by EDX data. By correlating SIMS ratio to known concentration, one can obtain absolute (not just relative) quantification. However, the difference in the information depths for the X-ray generation and secondary ion generation needs to be addressed. Therefore, we developed a diffusion couple based approach to correlate EDX and SIMS results.

In this presentation, we will summarize the development work and present our new results obtained from case studies in correlative analysis using real-world samples from Li ion batteries (Figure 2) and 
solar cell research. We will also highlight advanced methods for image correlation and image fusion using in-situ and ex-situ [3, 4] analysis to derive deeper insights from the multimodal datasets [5].

\section{References:}

[1] T. Wirtz et al, Nanotechnology 26 (2015), p. 434001.

[2] L. Yedra et al, Sci. Rep. 6 (2016), p. 28705.

[3] F. Vollnhals et al, Anal. Chem. 89 (2017), p. 10702.

[4] P. Kumar et al, Solar Energy Materials and Solar Cells 160 (2017), p. 398.

[5] This work is co-funded by FNR (Luxembourg) through project grant INTER/SNF/16/11536628. We acknowledge Andrew Kercher and Dr. Nancy Dudney at Oak Ridge National Laboratory, TN, USA for the synthesis of $\mathrm{LiV}_{3} \mathrm{O}_{8}$ thin films cathodes as a part of work supported by Center for Mesoscale Transport Properties, an Energy Frontier Research Center supported by the U.S. Department of Energy, Office of Science, Basic Energy Sciences, under award \#DE-SC0012673.
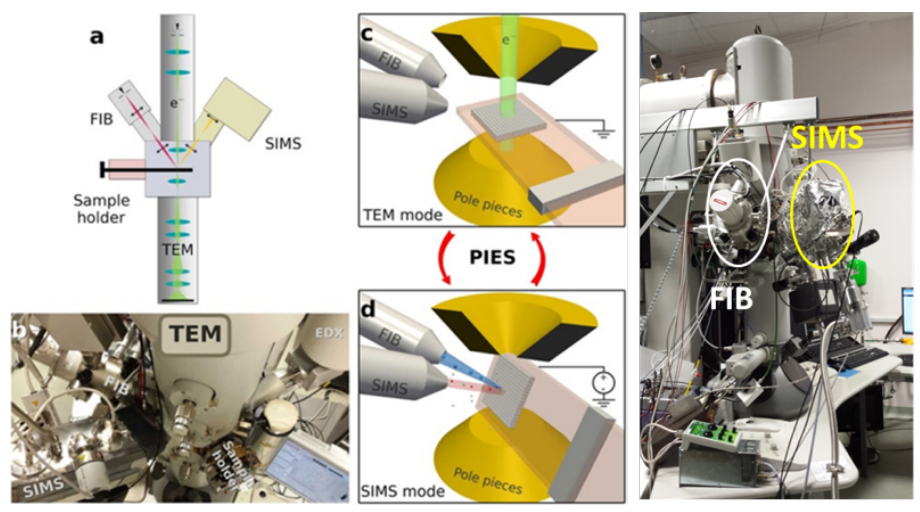

Figure 1. The layout and schematics of the in-situ TEM-SIMS instrument (Adapted from [2])

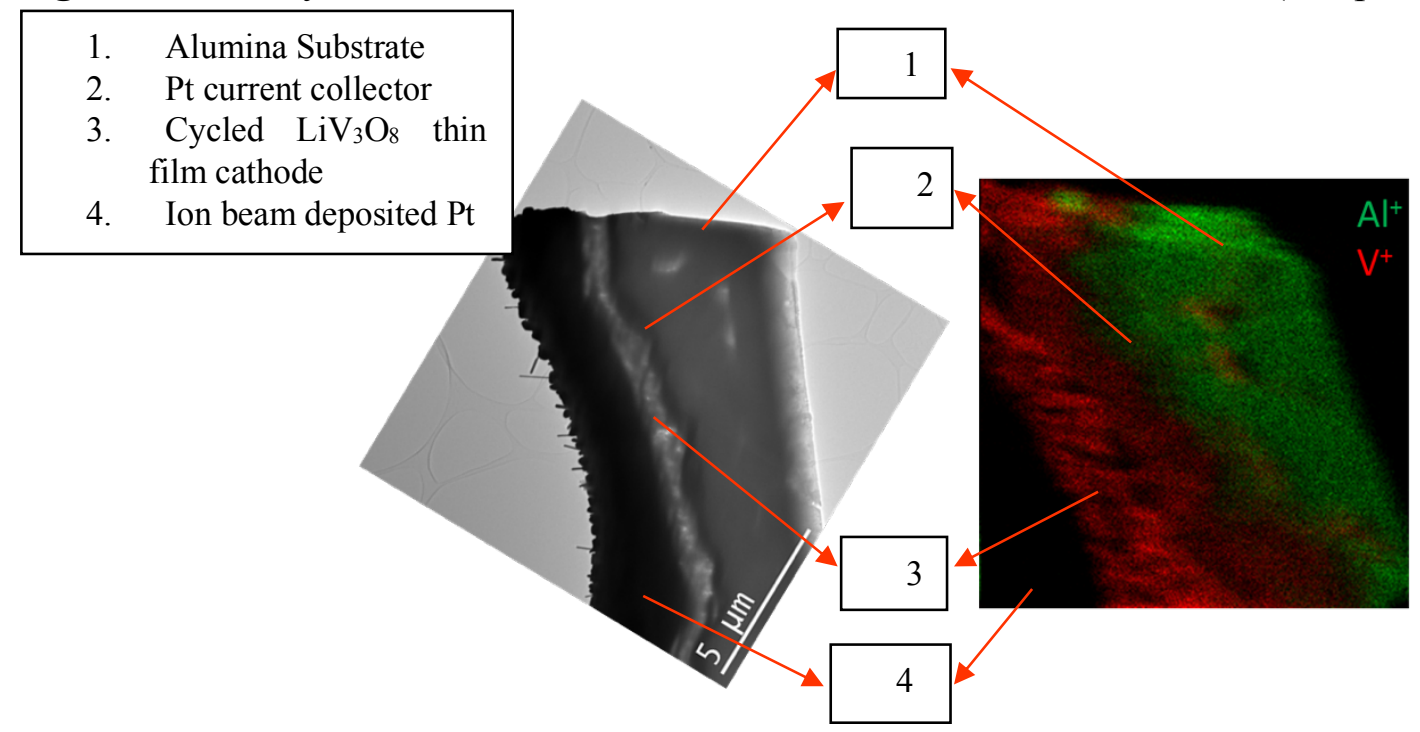

Figure 2. Bright-Field TEM and corresponding SIMS images (overlay of $\mathrm{Al}^{+}$and $\mathrm{V}^{+}$maps) from a cycled sputter deposited $\mathrm{LiV}_{3} \mathrm{O}_{8}$ thin film Li ion battery cathodes (initially annealed at $350 \mathrm{C}$ for $2 \mathrm{hrs}$, then100nm LiPON deposited on it) obtained from the in-situ TEM-SIMS instrument. The study focuses on understanding the vanadium dissolution in the cycled cathodes. 